\title{
Surgical Management of Drug Resistant Epilepsy
}

\author{
Mamoun Abo-Shousha1, Alaa Rashad Ibrahim ${ }^{1}$, Ahmed Mohamed Ali Gabr ${ }^{1, *}$
}

ABSTRACT

*CorrespondingAuthor:

Ahmed Gabr

ahmed_asyot2010@yahoo.com

Received for publication December 29, 2019; accepted Januaryl6, 2020; Published on line January 22, 2020.

Copyright 2020 The Authors published by Al-Azhar University, Faculty of Medicine, Cairo, Egypt. All rights reserved. This an openaccess article distributed under the legal terms, where it is permissible to download and share the work provided it is properly cited. The work cannot be changed in anyway or used commercially.

doi: 10.21608/aimj.2020.21691.1040

${ }^{1}$ Neuro Surgery Department, Faculty of Medicine, Al-Azhar University Cairo, Egypt.

Background: Seizure is a chronic brain disease that is caused by various factors and characterized by recurrent, episodic and temporary central nervous system dysfunction which results from excessive discharge of brain neurons.

Objectives: This work aims to review surgical outcome for drug resistant epilepsy of different causes to identify which patient would get the most benefit.

Patients and Methods: Prospective and retrospective study on 20 cases with drug resistant epilepsy at Al-Azhar university hospitals.

Results: The study showed, for the first time that after one year follow up, $58 \%$ of patients in the ATL group was seizure- free compared to only $8 \%$ in the medical therapy group $(p<0.001)$. Further, those in the surgical group had less frequent focal seizures and significantly better quality of life compared to the non-surgical group ( $p<0.001$ for both comparisons). In the surgical group 4 patients $(10 \%)$ had mild language and memory deficits against one dead in the non-surgical group.

Conclusion: The objective of epilepsy surgery is complete seizure control without any resulting post-surgical functional deficits.

Not all people who have done epilepsy surgery are seizure free following surgery, but there is usually a significant decrease in seizures. Some people may have some seizures immediately after surgery but this doesn't mean that the surgery wasn't successful.
\end{abstract}

Keywords: Surgical Management, drug resistant epilepsy, lesionectomy.

\begin{abstract}
Disclosure: The authors have no financial interest to declare in relation to the content of this article. The Article Processing Charge was paid for by the authors.

Authorship: All authors have a substantial contributions to the article.
\end{abstract}

\section{INTRODUCTION}

Epilepsy (ILAE2014): is a disease of the brain defined by any of the following conditions: at least 2 unprovoked (or reflex) seizures occurring more than 24 hours apart; one unprovoked (or reflex) seizure with relatively high recurrence risk (i.e., $\geq 60 \%$ ), occurring over the next 10 years or diagnosis of an Epilepsy syndrome. Seizure is a chronic brain disease that is caused by various factors and characterized by recurrent, episodic and temporary central nervous system dysfunction which results from excessive discharge of brain neurons. ${ }^{5}$

Drug-resistant epilepsy may be defined by International League Against Epilepsy (ILAE)as failure of adequate trials of two tolerated and appropriately chosen and used AED schedules (whether as monotherapies or in combination) to achieve sustained seizure freedom. ${ }^{7}$

As a serious health problem worldwide, epilepsy accounts for $1 \%$ of the world's diseases. ${ }^{10}$

Despite appropriate trials of at least two antiepileptic drugs, about a third of patients with epilepsy remain drug resistant (intractable; refractory). ${ }^{1}$
Traditionally, therapeutic failure of three antiseizure drugs defined intractability. ${ }^{6}$

Epilepsy surgery offers a potential cure or significant improvement to those with focal onset drug-resistant seizures. Unfortunately, epilepsy surgery is still underutilized which might be in part because of the complexity of pre-surgical evaluation. Pre-surgical evaluation includes classifying the seizure type, lateralizing and localizing the seizure onset focus (epileptogenic zone), confirming the safety of the prospective brain surgery in terms of potential neurocognitive deficits (language and memory functions), before devising a surgical plan.

Pathogenesis of drug-resistant epilepsy

The pathogenesis of epilepsy is complex and, at present, commonly acknowledged to be caused by the excitatory and inhibitory imbalance of the central nervous system. So far, the hypotheses regarding the pathogenesis of drug-resistant epilepsy mainly include:

\section{1- Transporter hypothesis}

Most antiepileptic drugs play the antiepileptic role in the brain via the blood-brain. ${ }^{8}$

Moreover, the overexpression of multidrug transporters that have a role in the efflux from the capillary endothelial cells which form the bloodbrain barrier may lead to increased intracellular drug efflux or isolated vesicles, resulting in decreased intracellular drug concentration or changed drug distribution; consequently, antiepileptic drugs in epileptogenic zone and surrounding tissues cannot achieve the effective drug concentration, which leads to drug resistance. ${ }^{9}$ 


\section{2- Target hypothesis}

This hypothesis proposes that antiepileptic drugs cannot inhibit the excessive discharge of neurons through binding the predetermined target when the structure or function of the target of antiepileptic drugs changes, resulting in uncontrollable epilepsy attack, which is mainly reflected by abnormal ion channel function. ${ }^{12}$

\section{Surgical treatment of drug-resistant epilepsy}

Although there were multiples of new-type antiepileptic drugs that have been continued to be approved for application, there still exist more than 30 percent of patients with epilepsy developed into drug-resistant epilepsy. ${ }^{1}$

With the continuous development of related science and technology, a variety of different surgical treatment methods emerged in response to the proper time and conditions, and now surgical treatment has become a very important therapeutic tool for drugresistant epilepsy treatment. The goal of surgery is to reduce the seizures, to avoid the adverse reaction after surgery, and to a certain extent, to improve the quality of life. In general, we need to adjust the preoperative state of patients to meet the criteria of surgery. At present, surgical treatment methods for epilepsy are divided into resective surgery, palliative surgery, neuromodulation and other surgical interventions.

The work aims to review surgical outcome for drug resistant epilepsy of different causes to identify which patient would get the most benefit.

\section{PATIENTS AND METHODS}

Prospective and retrospective study on 20 cases with drug resistant epilepsy at Al-Azhar university hospitals. All cases are subjected to history and clinical examination. (Clearance of epilepsy seminology).

-Investigation by MRI brain, EEG and CT brain.

-Surgical techniques: resective (Lesionectomy) Surgery, Corpus Callosotomy

Outcome: functional outcome, control of epilepsy, complications

Inclusion Criteria: Age: 10-50 years, all cases of epilepsy that are not controlled on 2 antiepileptic drugs (drug resistant) and all cases that cannot tolerate side effects of antiepileptics.

Exclusion Criteria: All cases that are controlled on two or less antiepileptic drugs without intolerable side effects and ages younger than 10 or older than 50 years

Statistical analysis:_Recorded data were analyzed using the statistical package for social sciences, version 20.0 (SPSS Inc., Chicago, Illinois, USA). Quantitative data were expressed as mean \pm standard deviation (SD). Qualitative data were expressed as frequency and percentage.

\begin{tabular}{|l|c|c|}
\hline \multicolumn{1}{|c|}{ Age (years) } & No. & \% \\
\hline$<15$ years & 12 & $60.0 \%$ \\
\hline $15-20$ years & 3 & $15.0 \%$ \\
\hline$>20$ years & 5 & $25.0 \%$ \\
\hline Total & 20 & $100.0 \%$ \\
\hline
\end{tabular}

Table (1): Distribution of the study group according to their age $(n=20)$.

This table shows that the $<15$ years $(60.0 \%), 15-20$ years $(15.0 \%)$ and $>20$ years $(25.0 \%)$ of age (years), also ranged age $10-36$ with mean $17.30 \pm 9.20$

\begin{tabular}{|l|c|c|}
\hline \multicolumn{1}{|c|}{ Sex } & No. & \% \\
\hline Female & 10 & $50.0 \%$ \\
\hline Male & 10 & $50.0 \%$ \\
\hline Total & 20 & $100.0 \%$ \\
\hline
\end{tabular}

Table (2): Distribution of the study group according to their sex $(n=20)$.

This table shows that the female (50\%) and male (50\%) of sex.

\begin{tabular}{|l|c|}
\hline \multicolumn{1}{|c|}{ Duration of disease (years) } & No. \\
\hline Range & $1-8$ \\
\hline Mean \pm SD & $3.60 \pm 2.28$ \\
\hline Median (IQR) & $3(3)$ \\
\hline
\end{tabular}

Table (3): Distribution of the study group according to their duration of disease

This table shows that the ranged 1-8 with mean $3.60 \pm 2.28$ and median 3 (3) of duration of disease

\begin{tabular}{|l|c|c|}
\hline \multicolumn{1}{|c|}{ Semiology of epilepsy } & No. & \% \\
\hline Generalized tonic colonic fits & 15 & $75.0 \%$ \\
\hline Left focal tonic colonic fits of upper limb \& lower limb & 2 & $10.0 \%$ \\
\hline Right focal fits of upper limb \& lower limb with secondary generalization & 2 & $10.0 \%$ \\
\hline
\end{tabular}

Table (4): Distribution of the study group according to their semiology of epilepsy ( $n=20)$.

This table shows that the generalized tonic colonic fits (75.0\%), Left focal tonic colonic fits of upper limb \& lower limb (10.0\%) and Right focal fits of upper limb \& lower limb with secondary generalization (10.0\%) of semiology of epilepsy. 


\begin{tabular}{|l|c|c|}
\hline \multicolumn{1}{|c|}{ MRI findings } & No. & \% \\
\hline Normal & 2 & $10.0 \%$ \\
\hline Right Parietal arachnoid cyst & 2 & $10.0 \%$ \\
\hline Right insular dysplasia & 2 & $10.0 \%$ \\
\hline Left temporoparietal arachnoid cyst & 3 & $15.0 \%$ \\
\hline Left temporal Oligoclendroglioma grade II9 & 2 & $10.0 \%$ \\
\hline Left temporal Xanthoastrocytoma grade II & 2 & $10.0 \%$ \\
\hline Left temporal low grade glioma & 2 & $10.0 \%$ \\
\hline Left temporal Ganglioglioma grade I & 2 & $10.0 \%$ \\
\hline Right temporal encephalomalacia & 1 & $5.0 \%$ \\
\hline Right temporal arachnoid cyst & 2 & $10.0 \%$ \\
\hline Total & 20 & $100.0 \%$ \\
\hline
\end{tabular}

Table (5): Distribution of the study group according to their MRI findings ( $\mathrm{n}=20)$.

This table shows that the Normal (10.0\%), Right Parietal arachnoid cyst (10.0\%), Right insular dysplasia (10.0\%), Left temporoparietal arachnoid cyst (15.0\%), Left temporal Oligodendroglioma grade II (10.0\%), Left temporal Xanthoastrocytoma grade II (10.0\%), Left temporal low grade glioma (10.0\%), Left temporal ganglioglioma grade I (10.0\%), Right temporal encephalomalacia (5.0\%) and Right temporal arachnoid cyst (10.0\%) of MRI findings.

\begin{tabular}{|l|c|c|}
\hline \multicolumn{1}{|c|}{ EEG } & No. & \% \\
\hline Right centroparietotemporal epileptic discharge & 4 & $20.0 \%$ \\
\hline Bilateral temporal epileptogenic activity with tendency to generalization & 2 & $10.0 \%$ \\
\hline Left temporal epileptic discharge & 9 & $45.0 \%$ \\
\hline Normal & 3 & $15.0 \%$ \\
\hline Right occipitoparietal epileptogenic activity & 2 & $10.0 \%$ \\
\hline Total & 20 & $100.0 \%$ \\
\hline
\end{tabular}

Table (6): Distribution of the study group according to their EEG $(\mathrm{n}=20)$.

This table shows that the Right centroparietotemporal epileptic discharge (20.0\%), bilateral temporal epileptogenic activity with tendency to generalization (10.0\%), Left temporal epileptic discharge (45.0\%), Normal (15.0\%) and Right occipitoparietal epileptogenic activity (10.0\%) of EEG.

\begin{tabular}{|l|c|c|}
\hline \multicolumn{1}{|c|}{ Antiepileptic drugs } & No. & \% \\
\hline Phynetion & 10 & $50.0 \%$ \\
\hline Levetiracetam & 16 & $80.0 \%$ \\
\hline Valproate & 10 & $50.0 \%$ \\
\hline Barbiturate & 2 & $10.0 \%$ \\
\hline Carbamazepine & 10 & $50.0 \%$ \\
\hline ClonaZepam & 2 & $10.0 \%$ \\
\hline Toperamate & 2 & $10.0 \%$ \\
\hline
\end{tabular}

Table (7): Distribution of the study group according to their antiepileptic drugs ( $\mathrm{n}=20)$.

This table shows that the Phynetion (50.0\%), Levetira cetam (80.0\%), Valproate $(50.0 \%)$, Barbiturate (10.0\%), Carbamazepine (50.0\%) and Clonazepam (10.0\%) of antiepileptic drugs.

\begin{tabular}{|l|c|c|}
\hline \multicolumn{1}{|c|}{ Antiepileptic drugs } & No. & \% \\
\hline Phenytoin \& Levetiracetam & 4 & $20.00 \%$ \\
\hline Phenytoin \& Levetiracetam \& Valproate & 2 & $10.00 \%$ \\
\hline Phenytoin \& Levetiracetam \& Valproate \& Clonazepam & 2 & $10.00 \%$ \\
\hline Phenytoin \& Levetiracetam \& Carbamazepine & 2 & $10.00 \%$ \\
\hline Levetiracetam \& Valproate & 2 & $10.00 \%$ \\
\hline Levetiracetam \& Carbamazepine & 2 & $10.00 \%$ \\
\hline Levetiracetam \& Carbamazepine \& Toperamate & 2 & $10.009 \%$ \\
\hline Valproate \& Barbiturate \& Carbamazepine & 2 & $10.00 \%$ \\
\hline Valproate \& Carbamazepine & 2 & $10.00 \%$ \\
\hline Total & 20 & $100.00 \%$ \\
\hline
\end{tabular}

Table (8): Distribution of the study group according to their antiepileptic drugs $(n=20)$.

This table shows that the Phenytoin \& Levetiracetam $20.00 \%$, Phenytoin \& Levetira cetam \& Valproate $10.00 \%$, Phenytoin \& Levetiracetam \& Valproate \& Clonazepam 10.00\%, Phenytoin \& Levetiracetam \& Carbamazepine $10.00 \%$, Levetiracetam \& Valproate $10.00 \%$, Levetiracetam \& Carbamazepine $10.00 \%$, Levetiracetam \& Carbamazepine \& Toperamate $10.00 \%$, Valproate \& Barbiturate \& Carbamazepine $10.00 \%$ and Valproate \& Carbamazepine $10.00 \%$ of Antiepileptic drugs. 


\begin{tabular}{|l|c|c|}
\hline \multicolumn{1}{|c|}{ Surgical intervention } & No. & \% \\
\hline Excision & 14 & $70.0 \%$ \\
\hline Excision \& fenestration of cyst to subarachnoid space & 2 & $10.0 \%$ \\
\hline Callosotomy & 4 & $20.0 \%$ \\
\hline Total & 20 & $100.0 \%$ \\
\hline
\end{tabular}

Table (9): Distribution of the study group according to their surgical intervention $(n=20)$.

This table shows that the Excision (70.0\%), Excision \& fenestration of cyst to subarachnoid space $(10.0 \%)$ and Callosotomy $(20.0 \%)$ of surgical intervention.

\begin{tabular}{|l|c|c|}
\hline \multicolumn{1}{c|}{ Engl's Class } & No. & $\%$ \\
\hline I & 12 & $60.0 \%$ \\
\hline II & 2 & $10.0 \%$ \\
\hline III & 2 & $10.0 \%$ \\
\hline IV & 4 & $20.0 \%$ \\
\hline
\end{tabular}

Table (10): Distribution of the study group according to their Engl's class ( $\mathrm{n}=20)$.

This table shows that the class I (60\%), class II (10\%), class III (10\%), class IV (20\%) of Engl's class.

\section{DISCUSSION}

Postsurgical outcome in epilepsy surgery is mainly assessed based on seizure-freedom or seizure reduction, neurocognitive functioning, and change in overall QOL. More detailed elements in measuring outcome include factors such as work eligibility, driving, etc. Of particular concern, are language, verbal \& memory function following temporal lobe surgeries.

Interestingly, despite the evidence supporting the efficacy and safety of epilepsy surgery, until 2001 there was no such evidence based on randomized controlled trials (RCT). The first RCT included 80 patients with TLE randomly assigned to surgery (Anterior Temporal Lobectomy), or continuing medical therapy, for one year to compare both seizure control outcome, and quality of life (QOL), disability, and mortality.

This study showed that after one year follow up, 58\% of patients in the ATL group were seizure- free compared to only $8 \%$ in the medical therapy group ( $p<0.001)$. Further, those in the surgical group had less frequent focal seizures and significantly better quality of life compared to the non-surgical group ( $p<$ 0.001 for both comparisons). In the surgical group 4 patients (10\%) had mild language and memory deficits against one dead in the non-surgical group. ${ }^{11}$ Since postsurgical outcome significantly depends on seizure type and seizure onset location, different types of epilepsies and surgical approaches are to be studied separately.

At our study, follow up of the cases along 1 year postoperatively showed 16 patient $(80 \%)$ showed improvement as regard the frequency of attacks, decrease in therapeutic dose of antiepileptic drugs, better psychosocial outcome, 12 of which $(60 \%)$ became seizure free.

If the patient is taking multiple antiepileptic drugs (AEDs), an attempt is made to reduce the doses to nontoxic (but effective) levels or to diminish the most poorly tolerated medication sometime after surgery. If the patient is seizure free after about 2 years, the provider may discuss with the patient the pros and cons of discontinuing multiple AEDs. Patients are strongly urged to continue medications for many years postoperatively; there is a 1 in 3 chance of having a seizure in the 5 years following medication withdrawal after epilepsy surgery.

At our study (5\%) who has undergone corpus Callosotomy was observed in the ICU for the first evening following surgery. During this time, neurologic parameters were fluctuating and complicated by the disconnection syndrome. The patient was not verbalize readily or respond quickly, which was improved gradually by rehabilitation and speech therapy.

By the second postoperative day, the patient's normal baseline neurological status began to return.

A 2011 study identified long-term outcomes of epilepsy surgery in adults. A cohort of 615 adults who underwent surgery was followed up for a median of 8 years. Five years after surgery, 52\% of patients remained seizure-free, apart from simple partial seizures (SPS). Patients who had anterior temporal resections were less likely to experience seizure recurrence than those who underwent extratemporal resections. The longer a patient was seizure free, the less likely the chance of relapse. ${ }^{2}$

The results of one randomized controlled study conclusively show that patients with medial temporal lobe epilepsy who have medication-resistant seizures (who have failed at least two AEDs approved for treatment of focal onset seizures at appropriate doses), even early on in the course of their epilepsy, benefit from antromedial temporal resection with regard to seizure outcome. ${ }^{3}$

One note of caution is that a proportion of those patients who underwent dominant temporal lobectomy had a statistically-significant decline in verbal function on neuropsychological testing. Several prior studies strongly suggested that the latter occurs over years with persistent complex partial seizures, with or without progression to tonic-clonic seizures. Therefore, delaying surgery may not prevent verbal deficits and likely exposes the patient to all the negative consequences of continued seizures. ${ }^{4}$

A study by Engel et al found that early surgical treatment may be beneficial. For patients who had mesial temporal lobe epilepsy and disabling seizures for no more than 2 consecutive years following adequate trials of 2 brand-name AEDs, resective surgery plus AED treatment resulted in a lower probability of seizures for at least 2 years post- 
treatment, as well as improved health-related quality of life, than continued AED treatment alone. ${ }^{4}$

Certain readily definable, surgically remediable syndromes are easily identified, have an excellent prognosis for seizure control, and have minimal surgical morbidity. Postoperatively, many patients with these syndromes have improved quality of life (QOL), improved cognitive, psychosocial, and occupational outcome. In children with catastrophic epilepsy due to diffuse hemispheric syndromes, early surgical intervention stops seizures and reverses the cognitive decline so that these patients can develop normally, eventually leading relatively normal lives.

\section{CONCLUSION}

The objective of epilepsy surgery is complete seizure control without any resulting post-surgical functional deficits, so pre surgical evaluation is to determine whether or not epilepsy surgery can be done and to define its chances of risk and benefit.

Surgery can be classified to curative procedures which are performed when tests consistently point to a specific area of the brain where the seizures begin, as the Temporal lobectomy which is the most common type of surgery, Cortical excision, Hemispherectomy and Palliative procedures are performed when a specific area in the brain can't be determined or when the seizure focus overlaps brain areas critical for movement as Vagus nerve stimulation. Not all people who have done epilepsy surgery are seizure free following surgery, but there is usually a significant decrease in seizures. Some people may have some seizures immediately after surgery but this doesn't mean that the surgery wasn't successful.

\section{REFERENCES}

1. Brodie MJ and Kwan P. Staged approach to epilepsy management. Neurology. 2002; 58(8):S2-8.

2. De Tisi J, Bell GS, Peacock JL, McEvoy AW, Harkness WF, Sander JW, et al. The long-term outcome of adult epilepsy surgery, patterns of seizure remission, and relapse: a cohort study. Lancet. 2011; 378(9800):1388-95.

3. Dwivedi R, Ramanujam B, Chandra PS, Sapra S, Gulati S, Kalaivani M, et al. Surgery for DrugResistant Epilepsy in Children. $N$ Engl J Med. 2017; 377 (17):1639-47.
4. Engel J, McDermott MP, Wiebe S, Langfitt JT, Stern JM, Dewar S, et al. Early surgical therapy for drug-resistant temporal lobe epilepsy: a randomized trial. JAMA. 2012; 307(9):922-30.

5. Fisher RS, Acevedo C, Arzimanoglou A, Bogacz A, Cross JH, Elger CE, Engel J, Forsgren L, French JA, Glynn M and Hesdorffer DC. ILAE official report: a practical clinical definition of epilepsy. Epilepsia. 2014; 55(4):475-82.

6. Kwan P and Brodie MJ. Early identification of refractory epilepsy. New England Journal of Medicine. 2000; 342(5):314-9.

7. Kwan P, Arzimanoglou A, Berg AT, Brodie MJ, Allen HW, Mathern G, Moshé SL, Perucca E, Wiebe $S$ and French J. Definition of drug resistant epilepsy: consensus proposal by the ad hoc task force of the ILAE commission on therapeutic strategies. Epilepsia. 2010; 51(6):1069-77.

8. Löscher $\mathrm{W}$, Luna-Tortós $\mathrm{C}$, Römermann $\mathrm{K}$ and Fedrowitz M. Do ATP-binding cassette transporters cause pharmacoresistance in epilepsy? Problems and approaches in determining which antiepileptic drugs are affected. Curr. Pharm. Des. 2011; 17(26):280828.

9. Margineanu DG and Klitgaard H. Mechanisms of drug resistance in epilepsy: relevance for antiepileptic drug discovery. Expert Opin. Drug Discov. 2009; 4(1):23-32.

10. Murray GJ. Global Comparative Assessments in the Health Sector: Disease Burden.Expenditure, Intervention Packages; 1994, Curr Neuropharmacol. 2018 Jan.

11. Wiebe S, Blume WT, Girvin JP and Eliasziw M. Effectiveness and Efficiency of Surgery for Temporal Lobe Epilepsy Study Group A randomized, controlled trial of surgery for temporal lobe epilepsy. N. Engl. J. Med. 2001; 345:311-18.

12. Koubeissi M. Neuropathology of the blood-brain barrier in epilepsy: support to the transport hypothesis of pharmacoresistance. Epilepsy Curr. 2013;13(4):169-71.

[http://dx.doi.org/10.5698/1535-7597-13.4.169]. [PMID: 240094] 